\title{
COVID-19 and Heart Disease
}

\author{
Masoud Eslami ${ }^{1}$ and Reza Mollazadeh (iD ${ }^{1, *}$ \\ ${ }^{1}$ Cardiology Department, Imam Khomeini Hospital Complex, Tehran, Iran \\ "Corresponding author: Associate Professor of Cardiology, Cardiology Department, Imam Khomeini Hospital Complex, Tohid Sq., P.O. Box: 1419733141, Tehran, Iran. Email \\ mollazar@yahoo.com \\ Received 2020 April 20; Accepted 2020 April 21.
}

Keywords: Cardiovascular, Coronavirus, COVID-19, SARS-CoV-2

\section{Dear Editor,}

Since the outbreak of Coronavirus Disease 2019(COVID19) in Wuhan, China, in late December 2019 (1), it has spread worldwide and turned to a pandemic. In our country, Iran, the first documented (proven with polymerase chain reaction) was reported by the Ministry of Health in early February 2020 from Qom, a city near Tehran. Besides the wellknown respiratory symptoms and complications, there are recently a few reports of cardiovascular involvement. The interaction of the cardiovascular system and COVID19 can be categorized into four parts: (1) acute cardiac injury, (2) chronic cardiovascular damage, (3) worsening of pre-existing cardiovascular damage, and (4) Drug-induced proarrhythmic effects.

There are a few reports of myocarditis and acute heart failure caused by Severe Acute Respiratory Syndrome Coronavirus 2 (SARS-CoV-2) (2). This is not out of mind due to the similarities of SARS-CoV-2 to a previous virus in this family named Middle East Respiratory Syndrome-related Coronavirus (MERS-CoV). As known, MERS-CoV can cause acute myocarditis and heart failure. This is probably due to the direct invasion of myocytes by the virus through angiotensin-converting enzyme 2 receptors, or more probably due to inflammatory reactions caused by the host immune system and the cytokine storm in severe cases. This host-virus interaction may lead to myocardial injury, as manifested with the increase of cardiac biomarkers (cardiac troponins). The result would be the reduction of left ventricular ejection fraction and clinical heart failure. Arrhythmia and even sudden cardiac death may also occur.

Considering the novelty of SARS-CoV-2, we do not know whether permanent damage to the cardiovascular system occurs or not. Up to now, reports have shown that myocarditis and bradycardia (3) are reversible. We need further evaluation (e.g., endomyocardial biopsy, positron emission tomography, cardiac magnetic resonance imag- ing, etc.) and longer follow-ups of the patients to deduce whether SARS-CoV-2 causes permanent cardiac injury or not.

The worst scenario occurs among COVID-19 patients with known pre-existing cardiovascular disease. This group encompasses a wide spectrum of patients with different disease severity and cardiac reserve. Some patients just have cardiovascular risk factors (diabetes mellitus, hypertension, and obesity), but others have coronary artery disease, overt heart failure, and cardiomyopathy. The patients in the latter group have a limited cardiac reserve and are prone to the aggravation of heart failure symptoms, worsening of angina pectoris, or an increase of arrhythmia burden. More than morbidity is the obvious increased risk of mortality in this group (4).

The last but not the least interaction is the druginduced proarrhythmic effects. According to our national protocol, hydroxychloroquine is the cornerstone of COVID-19 treatment. Proarrhythmia (QT interval prolongation) is a well-known side effect of this drug, especially at high doses (5). There are reports of sudden cardiac death among COVID-19 patients that may be due to proarrhythmic effects of the antiviral drugs.

We are still at the beginning of the pathway to knowing the COVID-19 pathophysiology, its effects on different organs, and the most effective treatment.

\section{Footnotes}

Authors' Contribution: Reza Mollazadeh developed the original idea and the protocol, wrote the manuscript, and is a guarantor. Masoud Eslami contributed to manuscript writing.

Conflict of Interests: The authors declare no conflict of interests. 
Funding/Support: The authors declare no funding/support.

\section{References}

1. Wang D, Hu B, Hu C, Zhu F, Liu X, Zhang J, et al. Clinical characteristics of 138 hospitalized patients with 2019 novel coronavirus-infected pneumonia in Wuhan, China. Jama. 2020.

2. Dong N, Cai J, Zhou Y, Liu J, Li F. End-stage Heart Failure with COVID-19: Strong Evidence of Myocardial Injury by 2019-nCoV. JACC: Heart Failure. 2020.
3. Azarkish M, Laleh Far V, Eslami M, Mollazadeh R. Transient complete heart block in a patient with critical COVID-19. Eur Heart J. 2020. doi: 10.1093/eurheartj/ehaa307. [PubMed: 32285920].

4. Zhou F, Yu T, Du R, Fan G, Liu Y, Liu Z, et al. Clinical course and risk factors for mortality of adult inpatients with COVID-19 in Wuhan, China: a retrospective cohort study. The Lancet. 2020.

5. Gautret P, Lagier J, Parola P, Meddeb L, Mailhe M, Doudier B, et al. Hydroxychloroquine and azithromycin as a treatment of COVID-19: results of an open-label non-randomized clinical trial. International Journal of Antimicrobial Agents. 2020:105949. 